\title{
Is ethnicity linked to incidence or outcomes of covid-19?
}

\author{
Preliminary signals must be explored urgently
}

\author{
Kamlesh Khunti professor of primary care diabetes and vascular medicine ${ }^{1}$, Awadhesh Kumar Singh \\ senior consultant ${ }^{2}$, Manish Pareek associate clinical professor in infectious diseases ${ }^{3}$, Wasim Hanif \\ professor of diabetes ${ }^{4}$
}

${ }^{1}$ Diabetes Research Centre, Leicester General Hospital, University of Leicester, Leicester, UK; ${ }^{2}$ Department of Endocrinology, G D Hospital and Diabetes Institute, Kolkata, India; ${ }^{3}$ Department of Infection and HIV Medicine, University Hospitals Leicester NHS Trust, Leicester, UK; ${ }^{4}$ Department of Diabetes and Institute of Translational Medicine, University Hospital Birmingham, Birmingham, UK

The novel disease covid-19, caused by severe acute respiratory syndrome coronavirus SARS-CoV-2, is now a pandemic with devastating implications for populations, healthcare systems, and economies globally. Systematic reviews of ethnically homogenous cohorts from China suggest that the key risk factors for hospital admission include age, male sex, and comorbidities such as cardiovascular disease, hypertension, and diabetes. ${ }^{1}$ The UK is the first country in the covid-19 surge with an ethnically diverse population and can therefore contribute to our understanding of the disease's effects in different ethnic groups, particularly those of South Asian or African Caribbean heritage. The ethnic minority population of the UK was around $13 \%$ at the time of the last census in 2011.

\section{Warning signs}

Concerns about a possible association between ethnicity and outcome were raised after the first 10 doctors in the UK to die from covid-19 were identified as being from ethnic minorities. ${ }^{3}$ These concerns were confirmed by observational data from the Intensive Care National Audit and Research Centre, showing that a third of covid-19 patients admitted to critical care units are from an ethnic minority background. ${ }^{4}$ Of 2249 patients admitted to 201 critical care units in England, $64.8 \%$ were white, $13.8 \%$ were Asian, $13.6 \%$ were black, and $7.8 \%$ were from other or mixed ethnic groups. Patients had a mean age of 60.1 years. Importantly these unadjusted descriptive data take no account of factors other than ethnicity that could influence the risk of critical care admission.

Ethnic minority groups have also been disproportionately affected by covid-19 in the US, highlighting potential racial, economic, and other inequalities. ${ }^{5}$ An analysis by the Washington Post reports that that counties with black majorities have three times the rate of covid-19 cases, and almost six times the rate of deaths, compared with counties where white residents are in the majority. ${ }^{6}$ The US experience may be influenced, however, by the lack of universal healthcare, unlike the UK, and caution is required when extrapolating across different health systems.

To get a clearer picture of ethnic disparities in incidence and outcome in the UK, we need detailed national data reported by ethnic group. This could be done through linking ethnicity data from Hospital Episode Statistics or Public Health England to mortality data from the Office of National Statistics. We welcome the announcement that the NHS and Public Health England will lead a review of the evidence on why ethnic minority populations seem to be disproportionately affected by covid-19.

\section{Potential reasons}

The higher observed incidence and severity in minority groups may be associated with socioeconomic, cultural, or lifestyle factors, genetic predisposition, or pathophysiological differences in susceptibility or response to infection. Possible susceptibilities include an increased risk of admission for acute respiratory tract infections, ${ }^{7}$ an increased prevalence of Vitamin D deficiency, ${ }^{8}$ vaccination policies in their country of birth and immune effects, ${ }^{9}$ increased inflammatory burden, and higher prevalence of cardiovascular risk factors such as insulin resistance and obesity than white populations. ${ }^{10}$ Some of these are also risk factors for increased disease severity in covid-19. ${ }^{11}$

Interest has also focused on the possibility of ethnic differences in the the expression of angiotensin converting enzyme 2 (the host receptor for SARS-CoV-2), ${ }^{12}$ and risk of both acute kidney injury ${ }^{13}$ and cardiac complications ${ }^{14}$ because of a higher prevalence of cardiovascular risk factors in ethnic minority populations. 
Ethnic minority communities are also more likely to be socioeconomically disadvantaged than white communities and often live in extended cohabiting families, potentially increasing the risk of virus transmission. Ethnic minorities in the UK and US have been shown to face several disadvantanges, including poor housing, overcrowding, and being more likely to be employed in low paid essential jobs, ${ }^{315}$ all of which make social distancing more challenging.

These suggestions require urgent exploration through robust analysis of routinely collected prospective data on covid-19, including age, sex, underlying morbidity, place of residence, area clustering, sociodemographic factors, laboratory measures, and burden of undiagnosed disease to determine if the observed signal between ethnicity and covid-19 outcomes is real or an artefact. Some of these data are already available. However, mixed methods research will be required to fully understand the complex interplay between the various biological, social, and cultural factors underlying these early findings.

Clear evidence to confirm or rule out an association between ethnicity and outcome in covid-19 is important not only for the UK but also for other regions such as South Asia and Africa, where the pandemic is at an earlier stage. Efforts must be coordinated to collect an international data set that includes ethnicity. If an association is confirmed, further research will be needed to determine the causes. Meanwhile, populations of all ethnicities must continue handwashing and hygiene, social distancing and self-isolation when required. The public should also be encouraged to maintain healthy lifestyles to optimise cardiometabolic and mental health. Finally, this important information must be communicated in an accessible and culturally appropriate way.

Competing interests: We have read and understood BMJ policy on declaration of interests and have no relevant interests to declare. KK is director of the Centre for Black Minority Health, University of Leicester. KK and WH are trustees of the charity South Asian Health Foundation and co-chairs of their Diabetes Working Group.
Provenance and peer review: Not commissioned, externally peer reviewed.

1 Li B, Yang J, Zhao F, etal . Prevalence and impact of cardiovascular metabolic diseases on COVID-19 in China. Clin Res Cardiol 2020. 10.1007/s00392-020-01626-9 32161990 Office of National Statistics, 2011 census. 2011.

3 Siddique $\mathrm{H}$. UK government urged to investigate coronavirus deaths of BAME doctors.Guardian 2020 Apr 10. https://www.theguardian.com/society/2020/apr/10/ukcoronavirus-deaths-bame-doctors-bma

4 Intensive Care National Audit and Research Centre. Covid-19 study case mix programme. 2020.

5 Resnick A, Galea S, Sivashanker K. Covid-19: the painful price of ignoring health inequities. BMJ Opinion, 18 Mar 2020. https://blogs.bmj.com/bmj/2020/03/18/covid-19-the-painfulprice-of-ignoring-health-inequities

6 Thebault $R$, Tran $A B$, Williams $V$. The coronavirus is infecting and killing black Americans at an alarmingly high rate. Washington Post 2020 Apr 7. https://www.washingtonpost. com/nation/2020/04/07/coronavirus-is-infecting-killing-black-americans-an-alarminglyhigh-rate-post-analysis-shows.

7 Simpson CR, Steiner MF, Cezard G, etal. SHELS researchers. Ethnic variations in morbidity and mortality from lower respiratory tract infections: a retrospective cohort study. $J$ R Soc Med 2015;108:406-17. 10.1177/0141076815588321 26152675

8 Martineau AR, Jolliffe DA, Hooper RL, etal . Vitamin D supplementation to prevent acute respiratory tract infections: systematic review and meta-analysis of individual participant data. BMJ 2017;356:i6583. 10.1136/bmj. 6658328202713

9 Miller A, Reandelar MJ, Fasciglione K, et al. Correlation between universal BCG vaccination policy and reduced morbidity andmortality for COVID-19: an epidemiological study. MedRxiv 2020.03.24.20042937 [Preprint.] 2020. 10.1101/2020.03.24.20042937. https:// www.medrxiv.org/content/10.1101/2020.03.24.20042937v1.full.pdf

10 Tillin T, Forouhi NG, McKeigue PM, Chaturvedi NSABRE Study Group. Southall And Brent REvisited: Cohort profile of SABRE, a UK population-based comparison of cardiovascular disease and diabetes in people of European, Indian Asian and African Caribbean origins. Int J Epidemiol 2012;41:33-42. 10.1093/ije/dyq175 21044979

11 CDC. Hospitalization rates and characteristics of patients hospitalized with laboratory-confirmed coronavirus disease 2019. https://www.cdc.gov/mmwr/volumes/69/ $\mathrm{wr} / \mathrm{mm} 6915 \mathrm{e} 3 . \mathrm{htm}$

12 Zhao, Y. Zhao Z, Wang Y, et al., Single-cell RNA expression profiling of ACE2, the putative receptor of Wuhan 2019-nCov. bioRxiv 2020.01.26.919985 [Preprint.] 2020. 10.1101/2020.01.26.919985.

$13 \mathrm{Xu} \mathrm{S}$, Fu L, Xiang HX, et al. Acute kidney injury at early stage as a negative prognostic indicator of patients with COVID-19: a hospital-based retrospective analysis. medRxiv 2020.03.24.20042408 [Preprint.] 2020. 10.1101/2020.03.24.20042408.

$14 \mathrm{Xu} \mathrm{H}$, et al. Acute myocardial injury of patients with coronavirus disease 2019. medRxiv 2020.03.05.20031591 [Preprint.] 2020. 10.1101/2020.03.05.20031591

15 Lymperopoulou K, Finney N. Socio-spatial factors associated with ethnic inequalities in dictricts of England and Wales, 2001-2011. Urban Stud 2017;54:2540-6010.1177/0042098016653725.

Published by the BMJ Publishing Group Limited. For permission to use (where not already granted under a licence) please go to http://group.bmj.com/group/rights-licensing/ permissions 